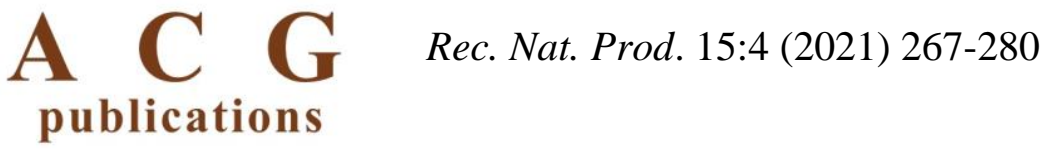

\title{
Understanding Radix Angelica sinensis Blood Replenishing mechanisms on Blood Deficiency Rats Based on a UPLC-Q/TOF-MS Metabolomics and Network Pharmacology
}

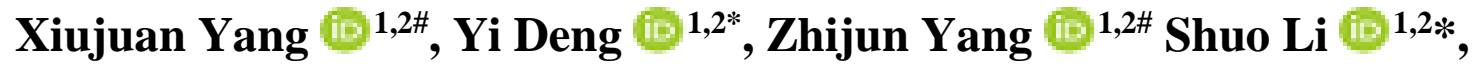 \\ Yunxiang Hai $\oplus^{1,2}$ and Yihong Tian $\left(^{1,2}\right.$
}

\author{
${ }^{I}$ Gansu University of Traditional Chinese Medicine, Gansu Province, Lanzhou 730000, P.R. China \\ ${ }^{2}$ Northwest Collaborative Innovation Center for Traditional Chinese Medicine, Gansu \\ Province, Lanzhou 730000, P.R. China
}

(Received January 28, 2021; Revised February 11, 2021; Accepted February 18, 2021)

\begin{abstract}
Radix Angelica sinensis (RAS) is a famous Chinese medicine with hematinic effects and has been applied for the treatment of blood deficiency syndrome for many years. Previous studies have indicated that RAS has beneficial effects in the treatment of hemolytic anemia. The hemolytic anemia/blood deficiency syndrome is a common syndrome that is often presented in most traditional Chinese medicine (TCM) clinics. Based on routine blood indicators, metabolomics analysis was conducted to investigate the mechanism of RAS in the treatment of hemolytic anemia. Multivariate and univariate statistical analysis were used to identify potential biomarkers in the serum. On administering RAS to the haemololitic anaemic rat, the levels of WBC, RBC, HGB, and PLT in AG tended to shift toward that of the control group. Additionally, all the 26 metabolites such as cholic acid, succinic acid and orotate which are regulated by blood deficiency appeared normal through the five metabolic pathways, such as linoleic acid metabolism, alanine, aspartic acid and glutamate metabolism, pyrimidine metabolism, arginine and proline metabolism. Thus, three metabolic pathways predicted by the network pharmacology were consistent with the metabolism pathway of Angelica sinensis: linoleic acid metabolism, arginine and proline metabolism, tryptophan metabolism. The integrated metabolomics and network pharmacology comprehensively improved the understanding of the physiological and metabolic state of an organism. The possible hematopoietic effects and underlying mechanism of action on hemolytic anemia rats after lavage with RAS water extracts, could potentially be elucidated by combining pharmacology with untargeted metabolomics. These pointed out the significance of metabolomics as a valuable tool for studying the essence of Chinese medicine's syndrome theory and the mechanism of RAS under anti-blood deficiency syndrome.
\end{abstract}

Keywords: Angelica Sinensis; replenishing blood effect; UPLC-Q/TOF-MS; metabolomics; network pharmacology. (C) 2021 ACG Publications. All rights reserved.

\section{Introduction}

Radix Angelica sinensis (RAS) is a famous Chinese medicine with hematinic effects, it has been applied for the treatment of blood deficiency syndrome for many years. Previous studies have indicated that RAS has beneficial effects in the treatment of hemolytic anemia. The hemolytic

\footnotetext{
* Corresponding authors:E-Mails: dengyi_gszy@163.com (Y. Deng); 290608323@qq.com (S. Li)

\# Equal first authors
} 
anemia/blood deficiency syndrome is a common syndrome that is often presented in most traditional Chinese medicine (TCM) clinics. Hemolytic anemia is a type of blood deficiency syndrome and its clinical manifestation is identified by the increased breakdown of erythrocytes. Moreover, acetyl phenyl hydrazine (APH), a strong oxidant, has a slow oxidative damaging effect on red blood cells [1]. RAS, which was first described in "Shennong's Classic of Materia Medica", is mainly produced in Gansu, China. However, only the roots of RAS can be used as a medicinal compound in TCM. According to the Chinese Pharmacopoeia, RAS has several beneficial effects on enriching blood, promoting blood flow, alleviating pain and relaxing the bowel [2,3]. Previous studies have shown that related indexes of liver and thymus are increased, index of spleen is decreased, and the levels of HGB, $\mathrm{RBC}$, and HGT are enhanced after treatment with RAS. This indicated that RAS improved peripheral blood indexes in mice with blood deficiency syndrome $[4,5]$.

In addition to genomics and proteomics, the emergence of metabolomics has provided deeper understanding of the occurrence and transformation of biometabolites and metabolic pathways within the living systems [6-8]. As novel methods for simultaneously imaging endogenous low molecular weight metabolites $(<1000 \mathrm{D})$ of complex biological samples through the development of quantitative and qualitative analyses, metabolomics has been applied to screen critical biomarkers and metabolic pathways in the fields of clinical diagnosis, treatment, nutritional biochemistry, and prognostic judgement of diseases [9]. In recent years, many studies that involves the application of metabolomics have been reported to reveal the principle of TCM syndrome and the efficacy of herbs, which are characterized as components with multi-targeting features [6-9]. A non-targeted metabolomics analysis process includes sample pretreatment, extraction of metabolites, full scan detection of LCMS, data pretreatment, statistical analysis, and metabolites structural identification that are based on UPLC-Q-TOF/MS [10,11].

The current study was therefore aimed at investigating the hematinic function of RAS and to explore the possible mechanisms involved in RAS metabolomics strategy. Moreover, the study was designed to analyze differential metabolite levels in the serum of rats before and after drug delivery using a high-resolution UPLC-Q-TOF/MS approach. Multivariate statistical analysis, including principal component analysis (PCA), partial least-squares-discriminant analysis (PLS-DA), and orthogonal partial least squares discriminant analysis (OPLS-DA), as well as univariate statistical analysis such as a student's T-test, were utilized for performing metabolomics analysis on rat serum samples. The screening of differential metabolite levels was conducted through metabolomics methods. Subsequently, differentially expressed metabolites were used for the Kyoto Encyclopedia of Genes and Genomes (KEGG) analysis pathways to identify the involved metabolic pathways and to compare the differences in metabolic pathways before and after RAS treatment.

\section{Materials and Methods}

\subsection{Material}

The LC-MS grade acetonitrile was purchased from Merck (Merck, Darmstadt, Germany). Ammonium acetate (HPLC/SPECTRO grade) was obtained from Sigma Aldrich (Sigma, USA, 70221). N-acetyl phenyl hydrazine (APH) was supplied by Shanghai middle qin chemical reagent co. LTD (Shanghai, China). All other reagents and chemicals were of analytical grade unless stated otherwise.

Angelica sinensis was purchased from Minxian county (Gansu Province, China), and was authenticated by Prof, Cheng Yi Li (Department of Pharmacy, Gansu University of Traditional Chinese Medicine, Lanzhou, China). Voucher specimens were deposited in the Herbarium of the Department of Pharmacy for Traditional Chinese Medicine of the Gansu University (Lanzhou, China).

\subsection{Sample Preparation}

Dried RAS (200 g) was extracted for 40 min. using boiling water of twelve times its quantity, filtered through gauze, and the procedure repeated twice on the dregs. The three filtrates were then combined and evaporated with rotary evaporation under a vacuum at $60{ }^{\circ} \mathrm{C}$ and concentrated to a 
volume of $200 \mathrm{~mL}$, which was equal to $1 \mathrm{~g} / \mathrm{mL}$ of crude herb drugs. The preparation was then stored at $4^{\circ} \mathrm{C}$ for further use.

\subsection{Animal and Drug Administration}

A total of 24 male Wistar rats (weighing between 220 to $250 \mathrm{~g}$ ) were obtained from the Animal experiment center of the Lanzhou University (Lanzhou, China). Rats were randomly divided into three groups ( $\mathrm{n}=8$ rats per group), including healthy control group (CG), hemolytic anemia model group (MG), and RAS group (AG). Each individual rat was housed separately. Rats were treated by oral gavage once a day for 12 consecutive days. Rats in the $\mathrm{MG}$ and the $\mathrm{AG}$ groups were hypodermically injected with $2 \%$ APH saline solution at doses of $100 \mathrm{mg} / \mathrm{kg}$ on day 1 and $50 \mathrm{mg} / \mathrm{kg}$ on day 4 . AG group were given $10.8 \mathrm{~g} / \mathrm{kg}$ body mass of RAS water extracts with $0.2 \mathrm{~mL}$ by oral gavage once every day for 12 consecutive days. Rats in CG and MG were given an equal volume of normal saline. All the drug administration procedures performed were as described in [12-14]. This research was approved by Animal Experimental Ethical Inspection of Gansu University of Chinese Medicine on date 01/03/2018 and approval number 2018-045.

\subsection{Biological Sample Collection}

Rats were administered with drugs for 12 consecutive days, and blood samples were sampled from the ocular vein. Rats were anesthetized with chloral hydrate $(300 \mathrm{mg} / \mathrm{kg})$ via intraperitoneal (i.p.) injection, after which blood was collected from the aorta abdominalis and plasma was prepared. Moreover, EDTA-containing plasma samples were collected for evaluation of RBC, HGB, HCT, WBC and PLT, whereas serum samples were collected for UPLC-Q-TOF/MS analysis.

For the collection of serum samples, rats $(n=8)$ were orally administered with RAS water extracts and anesthetized by ip injection of $10 \%$ aqueous chloral hydrate and subsequently blood was collected from the aorta abdominalis. Both the EDTA-containing plasma and serum were collected from the aorta abdominalis. Blood samples were then centrifuged for $15 \mathrm{~min}$ at $3000 \mathrm{rpm}$ at room temperature and the supernatant was then transferred to a clear centrifuge tube and centrifuged for 10 $\min$ at $12000 \mathrm{rpm}$ at $4^{\circ} \mathrm{C}$ to obtain serum. The serum samples were stored at $-80^{\circ} \mathrm{C}$ for UPLC-QTOF/MS analysis. Prior to pretreatment, serum samples $(100 \mu \mathrm{L}$ per tube) were thawed at room temperature and acetonitrile $(100 \mu \mathrm{L})$ was added to each sample. The mixture was then vortexed for 1 min and kept at $-20^{\circ} \mathrm{C}$ for $10 \mathrm{~min}$. A vacuum drying approach was applied to the supernatant, and the dry sample was dissolved in $100 \mu \mathrm{L}$ of acetonitrile water solution (acetonitrile: water $=1: 1$ ). The sample mixture was vortexed and centrifuged for $15 \mathrm{~min}$ at $14000 \mathrm{rpm}$ at $4{ }^{\circ} \mathrm{C}$, followed by mass spectrometry analysis. Moreover, $200 \mu \mathrm{L}$ supernatant was pooled from each sample for quality control (QC) purposes.

\subsection{Chromatographic and Mass Spectrometric Conditions}

Analyses were performed using an UHPLC (1290 Infinity LC, Agilent Technologies) coupled with a quadrupole time-of-flight (TOF, AB Sciex Triple TOF 6600, Foster City, USA). For HILIC separation, samples were analyzed using a $2.1 \mathrm{~mm} \times 100 \mathrm{~mm}$ ACQUIY UPLC BEH $1.7 \mu \mathrm{m}$ column (Waters, Ireland). In both the ESI positive and negative modes, the mobile phase consisted of: (A) 25 $\mathrm{mM}$ ammonium acetate and $25 \mathrm{mM}$ ammonium hydroxide in water; (B) acetonitrile. The gradient was $85 \% \mathrm{~B}$ for $1 \mathrm{~min}$, which was linearly reduced to $65 \%$ over $11 \mathrm{~min}$, then reduced to $40 \%$ in $0.1 \mathrm{~min}$ and kept for $4 \mathrm{~min}$. thereafter, it was increased to $85 \%$ in $0.1 \mathrm{~min}$, with a 5 -min re-equilibration period.

The ESI source conditions were set as follows: Ion Source Gas1 (Gas1) was set to 60, Ion Source Gas (Gas2) was set to 60, curtain gas (CUR) to was set to 30 , source temperature was $600^{\circ} \mathrm{C}$ and the Ion Spray Voltage Floating (ISVF) was $\sim 5500 \mathrm{~V}$. In the MS only acquisition, the instrument was set to acquire over the $\mathrm{m} / \mathrm{z}$ range of $60-1000 \mathrm{Da}$, and the accumulation time for the TOF MS scan was set at $0.20 \mathrm{~s} / \mathrm{spectra}$. Moreover, in the auto MS/MS acquisition, the instrument was set to acquire over the $\mathrm{m} / \mathrm{z}$ range of $25-1000 \mathrm{Da}$, and the accumulation time for the product ion scan was set at 0.05 s/spectra. The product ion scan was acquired using information dependent acquisition (IDA) with a 
selected high sensitivity mode. The collision energy (CE) was fixed at $35 \mathrm{~V} \pm 15 \mathrm{eV}$, and the declustering potential (DP) was set up $\pm 60 \mathrm{~V}$.

\subsection{Data Processing}

The raw MS data (wiff. scan files) were converted to MzXML files using Proteo Wizard MS Converter software and were processed using XCMS software for feature detection, retention time correction, and alignment. Metabolites were identified by accuracy mass $(<25 \mathrm{ppm})$ and MS/MS data that matched the database of our set standards. For extracted ion features, only variables containing more than $50 \%$ of nonzero measurement values in at least one group were kept. For the multivariate statistical analysis, SIMCA-P 14.1 software (Umetrics, Sweden) was used. After Pareto scaling, PCA, PLS-DA and OPLS-DA were performed. To evaluate the robustness of the model, the leave-one-out cross-validation as well as response permutation testing was used. Significantly different metabolites were identified based on the combination of a statistically significant threshold of variable influence on projection (VIP) values obtained from PLS-DA model and two-tailed Student's $t$ test ( $p$ value) on the raw data. Metabolites with VIP values larger than 1.0 and $\mathrm{p}$ values less than 0.01 were considered statistically significant. Single dimensional statistical analysis included that two-tailed Student's $t$ test ( $\mathrm{p}$ value) and variance analysis were also undertaken and the volcano plots drawn by the R software.

\subsection{Metabolite Identification and Pathway Analysis}

The human metabolome database (HMDB) (http://www.hmdb.ca/) and KEGG (www.genome.jp/kegg/) database were used to identify metabolites. In brief, experimental statistics were compared with data extracted from the online databases. Besides, MS/MS spectra and retention times were put into online database to validate the significant changes of metabolite levels. In addition, the Met PA web tool (http://www.metaboanalyst.ca) was applied as a metabolic enrichment analysis tool to visualize the effects on the pathways in the MG and AG groups [15].

\section{Results and Discussion}

The result on the effects of Radix Angelica sinensis on peripheral hemograms of hemolytic blood deficiency rats, metabolic profiles analysis through PCA and differential metabolites are provided in Figure 1 and Table 1. Based on this finding, when compared with the CG group, the levels of RBC, HGB, HCT and PLT in the MG showed a statistically significant decrease $(P<0.01)$, indicating that the model group was successfully established. Moreover, RBC, HGB, HCT and PLT levels in the AG group showed a statistically significant increase compared with the MG group $(P<$ 0.01 ), suggesting that treatment with RAS improved blood deficiency. The integrality and accuracy of data are essential for obtaining analytical results with statistical and biological significance. The integrality of data needs to be inspected, missing value needs to be deleted or marked and extreme values need to be excluded. It is recommended to normalize metabolomics data of all samples included in each group. In the original dataset, the "50" rule was applied to remove missing data [16]. After normalization of the total peak area, the data was processed by Pareto-scaling in SIMCA-P software. Subsequently, MS data were analyzed by multivariate statistics to discriminate between the MG and AG groups (Table 1. The relative standard deviation (RSD) $\leq 30 \%$ was used to select 3763 metabolites, ion numbers in the negative (ESI-) mode and 4048 in the positive (ESI+) mode.

The stability of the analytical approach is one of the most significant factors that affects the accuracy of metabolomics data. To confirm a stable system, the spectrograms of QC samples and PCA were compared. Moreover, during sample analysis, a pooled QC sample, which includes all analytes used in the experiment, was applied [17]. The UPLC-Q-TOF/MS was more suitable for metabolomics due to the higher resolution, peak capacity and sensitivity. Therefore, during UPLC-Q-TOF/MS analysis batch and to evaluate the stability of the instrument, QC samples were included in every eight samples [18] and its ion chromatograms were compared. When the intensity of the response and retention time of each chromatographic peak in QC samples overlapped, the instrument variation caused by error was relatively smaller. For all samples, the molecular features were extracted using XCMS software, and PCA was applied to analyze the distribution of metabolic profiles for the QC 
samples. Based on this finding, all QC samples clustered tightly in the PCA space, indicating sufficient repeatability, stability, and reliable experimental data. Moreover, significant differences were observed between the AG, MG and CG groups. The AG group showed a similar trend as the CG group.
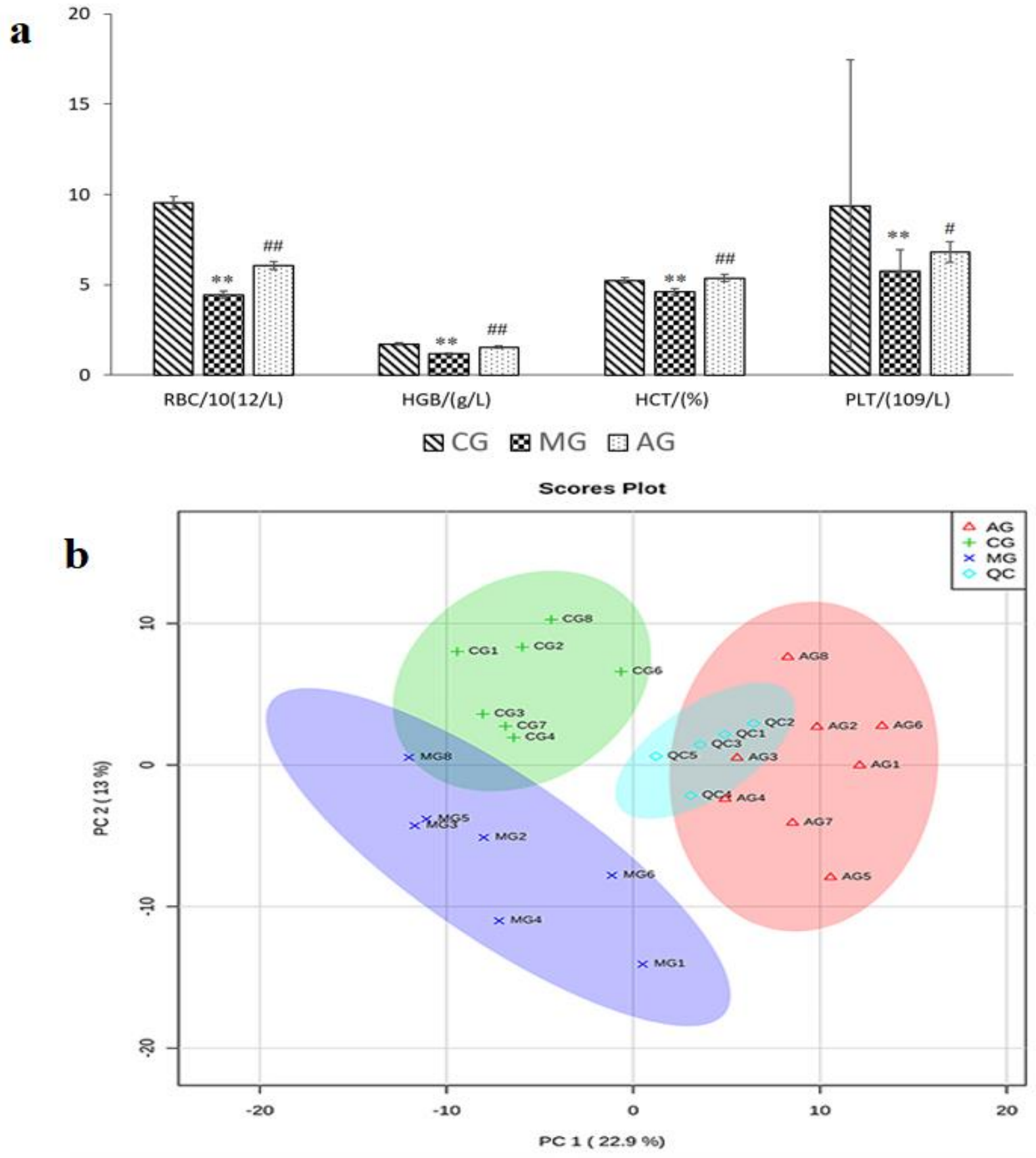

Figure 1. (a) Effects of Radix Angelica sinensis on peripheral hemograms $\left[\mathrm{RBC} / 10\left({ }^{12} / \mathrm{L}\right), \mathrm{HGB} /(\mathrm{g} / \mathrm{L})\right.$ $\left.\mathrm{x} 10^{2}, \mathrm{HCT} /(\%) \times 10^{1}, \mathrm{PLT} /\left(10^{9} / \mathrm{L}\right) \times 10^{2}\right]$ and $(\mathbf{b})$ metabolic profiles analysis through PCA ${ }^{* *} P<0.01$, compared with control group; ${ }^{\# \#} P<0.01$; compared with model group. 
Table 1. Differential metabolites in serum of MG and AG

\begin{tabular}{lllllll}
\hline \multicolumn{1}{c}{ Metabolites } & RT(s) & $\mathbf{m} / \boldsymbol{z}$ & VIP & FC & MG & AG \\
\hline L-Isoleucine & 501.12 & 130.09 & 2.33 & 0.73 & up & down \\
Creatine & 642.49 & 130.06 & 1.20 & 1.29 & up & up \\
Glycerophosphocholine & 724.96 & 258.11 & 3.77 & 0.80 & up & down \\
L-Pyroglutamic acid & 694.37 & 130.05 & 1.03 & 1.19 & down & up \\
Nicotinamide & 107.78 & 123.05 & 3.24 & 0.73 & up & down \\
L-Arginine & 950.24 & 173.11 & 1.62 & 1.62 & up & up \\
2'-Deoxyuridine & 196.96 & 457.16 & 2.07 & 0.38 & up & down \\
Linoleic acid & 70.74 & 279.23 & 17.18 & 0.72 & up & down \\
Uracil & 143.10 & 111.02 & 3.29 & 0.66 & up & down \\
Uridine & 285.83 & 243.06 & 3.11 & 0.62 & up & down \\
Cholic acid & 412.92 & 467.30 & 1.48 & 0.67 & up & down \\
Succinate & 742.17 & 117.02 & 1.04 & 0.67 & up & down \\
Orotate & 429.78 & 155.01 & 1.16 & 0.45 & up & down \\
3-Methylhistidine & 705.78 & 170.09 & 1.56 & 0.69 & up & down \\
1-Stearoyl-sn-glycerol-3-phosphocholine & 323.50 & 524.37 & 17.06 & 0.78 & down & up \\
Cytidine & 435.75 & 242.08 & 1.49 & 0.71 & up & down \\
Cytosine & 374.43 & 112.05 & 2.21 & 0.85 & up & down \\
L-Glutamine & 694.39 & 147.08 & 1.11 & 1.21 & down & up \\
L-Tryptophan & 468.18 & 203.08 & 2.63 & 1.38 & down & up \\
N6-methyladenosine & 231.72 & 282.12 & 1.16 & 0.60 & up & down \\
Allantoin & 327.13 & 157.04 & 2.09 & 0.80 & up & down \\
3-Indolepropionic acid & 196.19 & 190.08 & 1.27 & 0.62 & up & down \\
Nervonic acid & 65.41 & 365.34 & 1.67 & 0.50 & down & down \\
D-Ribose & 271.09 & 209.07 & 1.13 & 1.76 & down & up \\
Eicosapentaenoic acid & 76.08 & 303.23 & 2.07 & 1.24 & down & up \\
Deoxycytidine & 374.39 & 228.10 & 1.34 & 0.86 & up & down \\
\hline
\end{tabular}

s:second

\subsection{Multivariate Statistical Analysis Among All Groups}

Principle Component Analysis (PCA) score plots of the serum of rats in the CG, MG, and AG groups showed that the CG and AG groups were well separated from the model group, demonstrating significant changes in metabolic profiles in the hemolytic rat model. Data obtained from serum of rats in the AG group was similar to that of rats in the control group. PLS-DA and OPLS-DA are supervised statistical analysis methods, which establish the relationship between expression of metabolites and sample classification by partial least squares regression, and then predict the sample classification. Moreover, to determine how the expression pattern of each metabolite influences and interprets each group classification and discrimination, the variable importance for the projection (VIP) was calculated to auxiliary screen the sign metabolites (screening standard includes a VIP score > 1.0). PLS-DA score plots were established by the cross-validation parameters $\mathrm{R} 2 \mathrm{Y}$ and $\mathrm{Q} 2$, which represented the total explained variation for the $\mathrm{X}$ matrix and the predictability of the model. The validity of the model was explored by the R2Y and Q2 parameters in the PLS-DA model. R2Y estimated the sufficiency of fit of the model that represented the fraction of explained Y-variation, whereas Q2 estimated the ability of prediction. Reliable models were obtained when the cumulative values of R2Y and Q2 were above 0.8 [19]. As shown in Figure 2a-h, the quality parameters of PLS-DA score plots between rats in the AG and MG groups in positive and negative ion mode were as follows: $\mathrm{R} 2 \mathrm{Y}=0.995, \mathrm{Q} 2=0.914, \mathrm{R} 2 \mathrm{Y}=0.992, \mathrm{Q} 2=0.954$, demonstrating that the PLS-DA had sufficient predictive ability and reliability. Quality parameters of OPLS-DA score plots between rats in the AG and MG groups in positive and negative ion mode were as follows: $\mathrm{R} 2 \mathrm{Y}=0.999$, $\mathrm{Q} 2=0.638, \mathrm{R} 2 \mathrm{Y}=0.992, \mathrm{Q} 2=0.937$. 
a
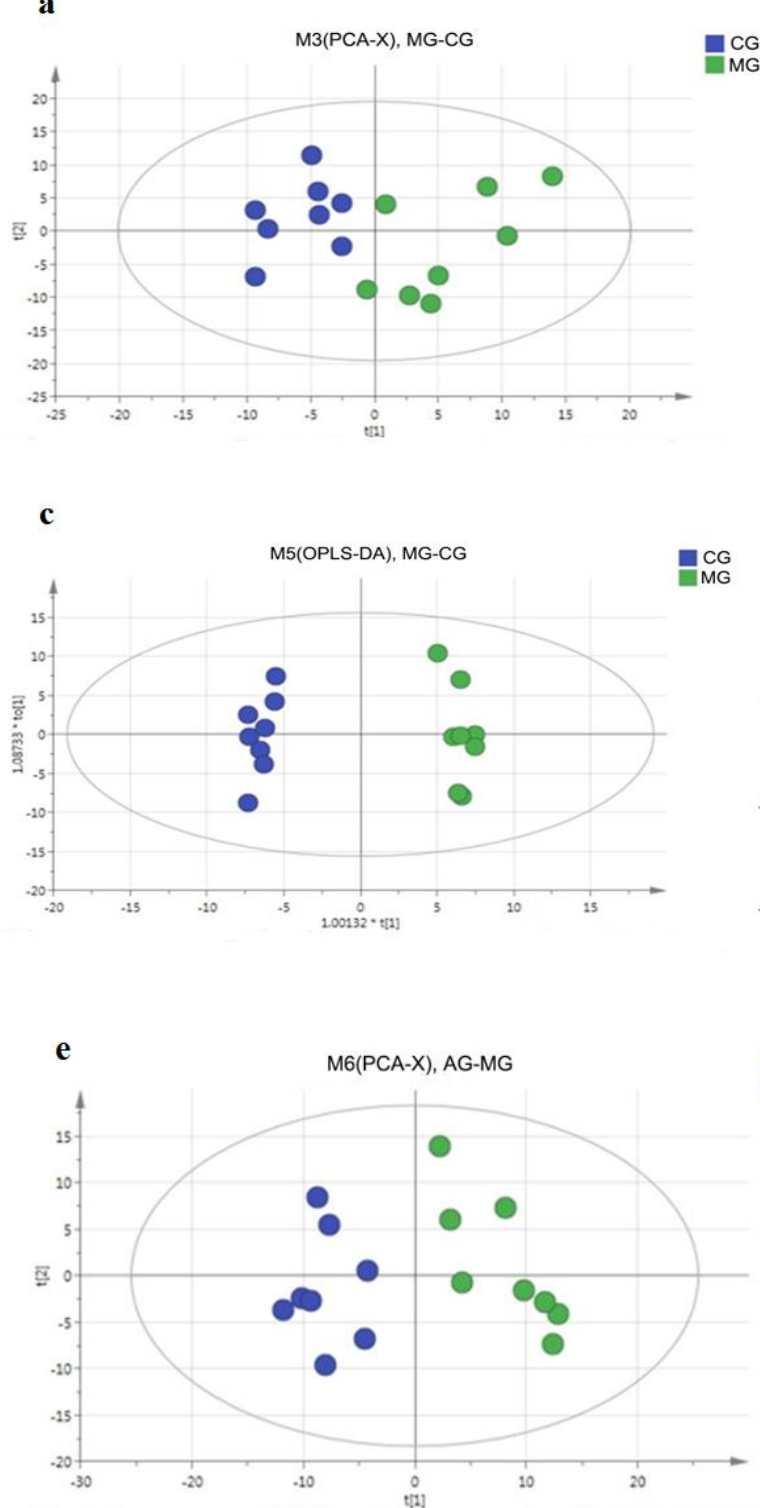

g

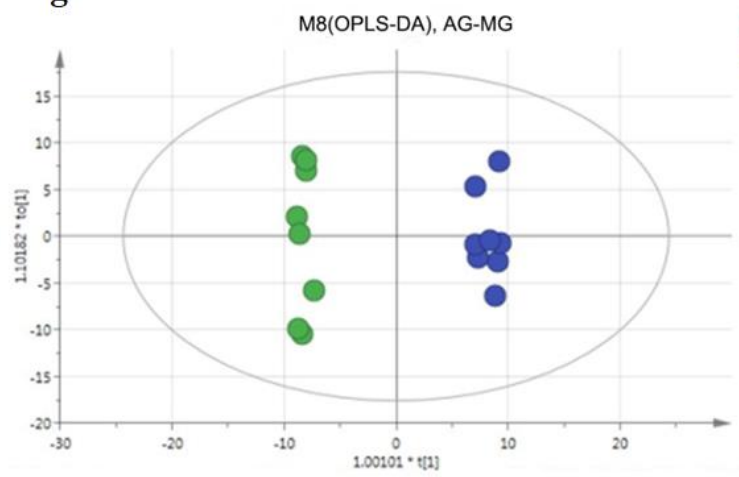

EMG

Aing

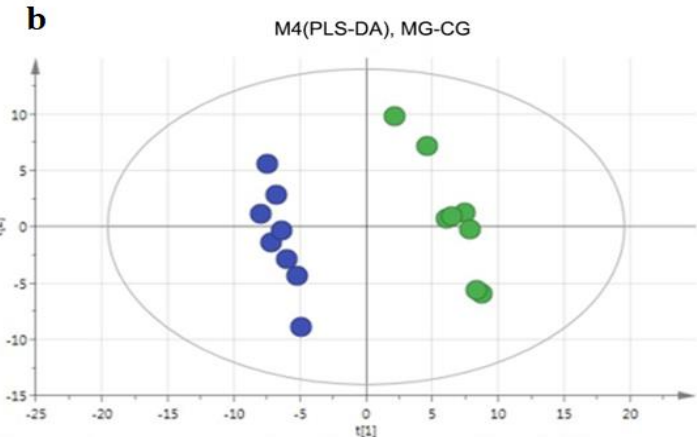

CG
MG

d

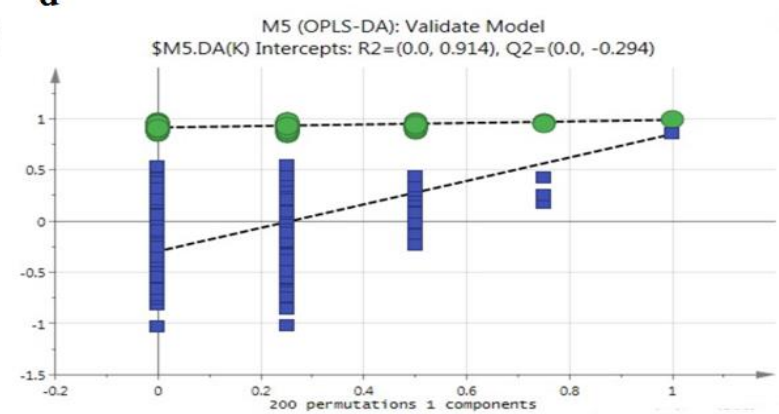

MG

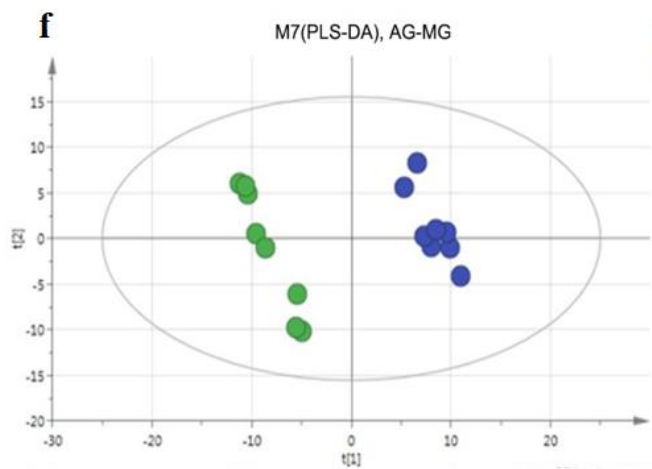

h
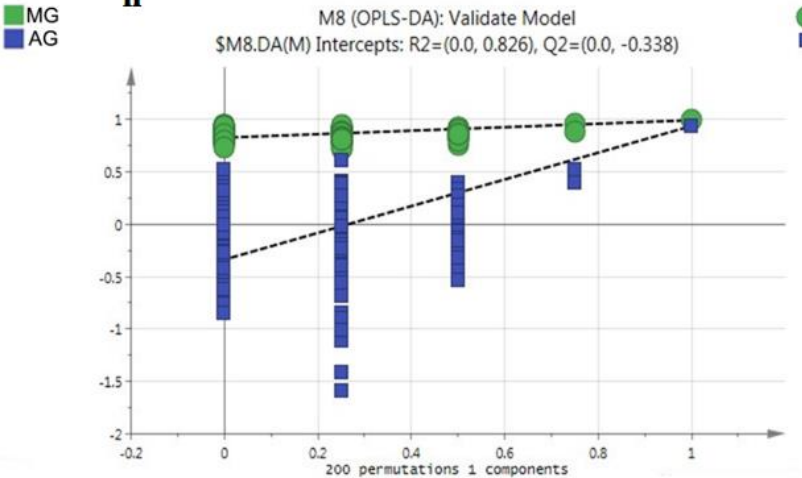

Figure 2. PLS-DA score plots between rats in the AG and MG groups in positive and negative ion mode

The significance of biomarkers was ranked using the VIP score (>1) from the PLS-DA model. For univariate analysis, candidate biomarkers were determined using Student's t-test statistics for comparison 
of the means between $\mathrm{AG}$ and $\mathrm{MG}$, and $\mathrm{MG}$ and $\mathrm{CG}$ groups. Values of $\mathrm{P}<0.05$ were considered statistically significant. After administering the RAS, 17 metabolites showed a remarkable decrease, whereas 6 metabolites significantly increased in the AG group. Based on their VIP scores, metabolites such as L-Pyroglutamic acid, 1-Stearoyl-sn-glycerol -3-phosphocholine, L-Glutamine, L-Tryptophan, D-Ribose, Eicosapentaenoic acid to increase in the AG group. The greater the VIP score of the metabolite, the more the contribution to the separation of sample classification, which classifies the metabolite as a potential biomarker. After treatment with RAS, the levels of the above-mentioned metabolites showed an opposite effect in the AG group.

a
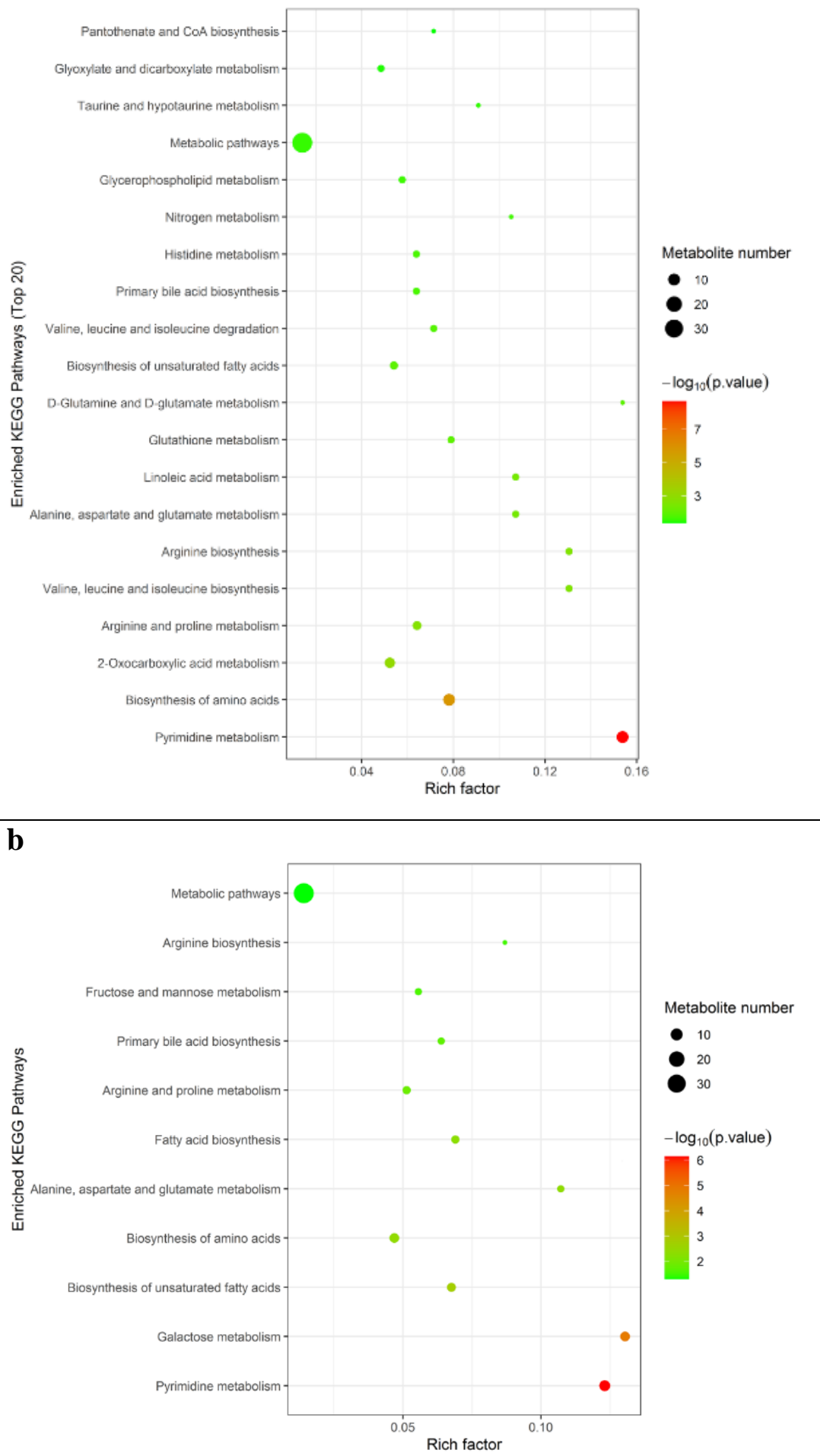

Figure 3. Metabolomic pathways in (a) Angelica sinensis group and in (b) blood deficiency group 


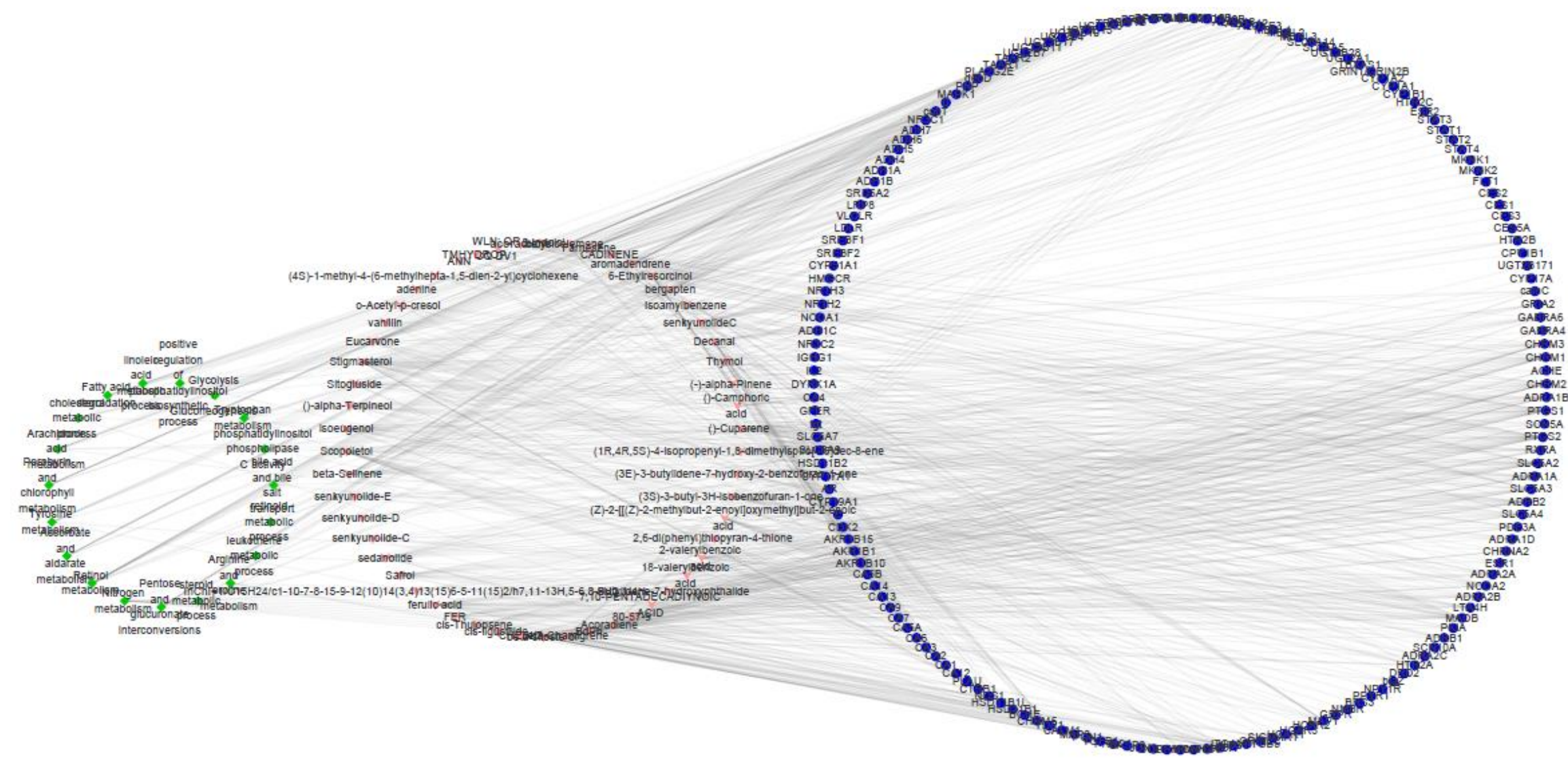

Figure 4. Components - target - Network passage 


\subsection{KEGG Pathway Analysis of Differential Metabolites}

Previous studies have shown that pathway analysis is an invaluable tool for understanding the relationships among genes and proteins [20]. The differentially expressed metabolites in the MG and AG groups were checked using the KEGG pathway database (http://www.genome,jp/kegg/) for their corresponding metabolic pathways. The result on the metabolomic pathways in Angelica sinensis and blood deficiency group is provided in Figure 3. The underlying mechanisms of action of RAS on hemolytic blood deficiency may be related to the linoleic acid metabolism, alanine, aspartic acid and glutamate metabolism, pyrimidine metabolism, arginine and proline metabolism.

\subsection{Angelica Network Pharmacology}

The network pharmacology information was based on the Chinese medicine system pharmacology analysis platform TCMSP (http://lsp.nwu.edu.cn/tcmsp.php), the PubChem Compound database (https://www.ncb i.nlm.nih.gov/pccompound) and other literature collection on Angelica active ingredient $[21,22]$. The potential targets of Angelica active ingredients were searched by TCMSP database platform, STITCH and Swiss Target Prediction, and KEGG pathway enrichment analysis of target proteins was performed by DAVID platform (https://david.ncifcrf.gov/) [23]. To clarify Angelica active ingredient, the relationship between the target and passage, Cytoscape3.2.1 software was used to create a "component target - Network passage" (Figure 4).

The "component-target- pathway" network of RAS was also constructed. The target predicted by network pharmacology was integrated with metabolites screened by metabolomics, and the integrated metabolic pathway map of Angelica sinensis was obtained as provide in Figure 5.

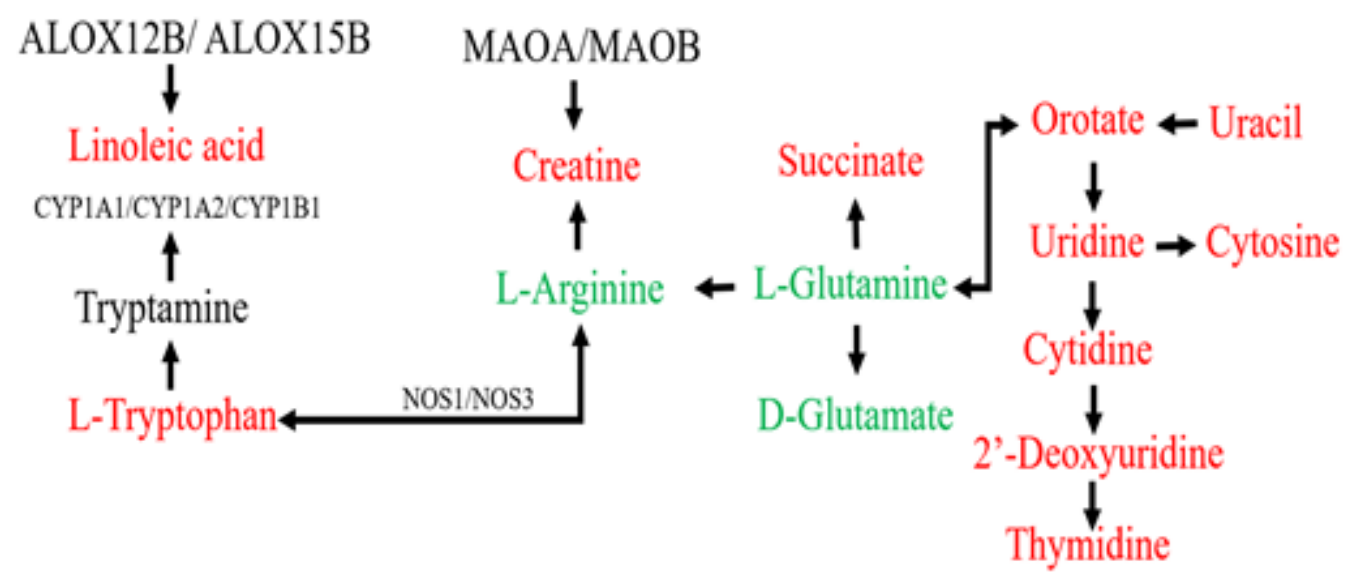

Figure 5. Metabolic pathway map of Angelica sinensis

\subsection{Discussion}

Anemia is a serious global public health concern that is associated with an increased risk of morbidity and mortality, especially in developing countries, and is characterized by a decrease in the hemoglobin content to a level that is less than $13 \mathrm{~g} / \mathrm{dL}$ in males or $12 \mathrm{~g} / \mathrm{dL}$ in females [24]. Anemia involves the decrease in the ability of blood to carry oxygen due to decrease in the total number of erythrocytes, a reduction in the concentration of hemoglobin per erythrocyte or a combination of both [25]. Hemolytic anemias are diseases that present with anemia due to the increased destruction of circulating abnormal RBCs, which can be classified into three major disorders, including membranopathies, hemoglobinopathies and enzymopathies [26]. Currently, three types of blood deficiency models are known. In this study, the hemolytic blood deficiency model, induced by APH, was used. APH is a strong oxidant that could slowly damage RBC by destroying RBC membrane proteins and lipids. Moreover, the RBC membrane could be dissolved and RBCs is disrupted [27,28].

The data obtained from the hemolytic anemia model used in this study was consistent with the blood deficiency syndrome in Chinese medicine. The model lasted for a relatively long time-period, which was sufficient to meet the evaluation of TCM efficacy. RAS is one of the most popular plants that is used in TCM for the treatment of anemia, constipation and cardiovascular disease, which has 
been used for thousands of years [29]. In addition, RAS produced by the Minxian county showed highest efficacy, best quality and highest yield [30]. RAS consists of many components, including coumarin, volatile oil, organic acid and polysaccharide [31,32]. Modern pharmacological studies indicated that RAS possessed a wide array of pharmacological activities, including anti-inflammatory, anti-cancer, anti-platelet aggregation, immune enhancement, anti-radiation damage and cardiovascular protective effect $[33,34]$.

Based on the study findings, it was demonstrated that the Angelica sinensis portrays a high potential in improving the blood deficiency syndrome of rats, and significantly increase the content of RBC, HGB and HCT in peripheral blood routine. Metabolomics were performed to investigate the enriching blood mechanism of RAS. All the 26 metabolites such as cholic acid, succinic acid and orotate which are regulated by blood deficiency moved towards normal and involved five metabolic pathways, such as linoleic acid metabolism, alanine, aspartic acid and glutamate metabolism, pyrimidine metabolism, arginine and proline metabolism. These always metabolites play a role in regulating the pyrimidine metabolism, which is considered one of the main metabolisms. Moreover, orotate, cytidine, uridine, 2'-deoxyuridine, cytosine and deoxycytidine are metabolites of the pyrimidine metabolism (http://www.genome,jp/kegg/). In the model group, the content of these metabolites increased, causing peripheral blood routine to be disordered. Compared with the MG group, the content of these metabolites in the AS group reduced significantly, indicating that RAS enriched the blood by regulating the pyrimidine metabolism. Indeed, it has been shown that the hemolytic anemia has a close relationship with abnormal metabolism of red cell nucleotides [35].

L-isoleucine and L-tryptophan are essential amino acids. Raw sugar and ketogenic amino acids are components of the RBC membrane. The $\alpha$-Keto acid can be exhaustively oxidized by the tricarboxylic acid cycle, which results in oxidative phosphorylation and ATP generation. Several studies have suggested that $\alpha$-keto acid could improve the HGB and RBC indexes of anemia in Chronic Kidney Disease (CKD) [36,37]. Moreover, the $\alpha$-keto acid can synthesize acetyl coenzyme A and participate in lipid metabolism. Tryptophan is metabolized via several pathways and acts as a precursor for the biosynthesis of the neurotransmitter serotonin (5-hydroxytryptamine, a biochemical messenger and regulator) [38]. The kynurenine pathway has continued to gain increasing attention because of its involvement in many diverse physiological and pathological processes [39,40]. Arginine is also involved in alpha-ketoglutarate metabolism. Compared with CG, L-isoleucine was increased, while arginine and L-tryptophan were significantly decreased. It has been reported that RBC membranes could be damaged by APH treatment and several amino acids on RBC membranes can also be changed [41]. There was an indication that the amino acids were disorganized, and after treatment with RAS, the levels of L-isoleucine were decreased, whereas the levels of arginine and Ltryptophan were significantly increased. The Levels of succinate were increased in serum of the MG group and after RAS treatment, it was reduced. Indeed, previous studies have also shown that succinate can lead to the disorder of Tricarboxylic Acid Cycle (TCA), if hypoxia is generated in an organism [42]. Furthermore, D-ribose is involved in the pentose phosphate pathway, which represents one way of oxidative decomposition of glucose [43]. Based on the present study, the D-ribose levels were decreased in the MG group, an indication that the pentose phosphate pathway could be inhibited. After the RAS treatment, the D-ribose levels were increased in the AG group. The L-pyroglutamic acid is known to be involved in the glutathione metabolism, which was also increased in the MG group, a condition that may cause toxic effects on the body [44]. Additionally, following RAS treatment, the L-pyroglutamic acid levels were decreased in the AG group.

Through this study, the mechanisms of hemolytic blood deficiency may be related with disorders of linoleic acid metabolism, alanine, aspartic acid and glutamate metabolism, pyrimidine metabolism, arginine and proline metabolism. The "component-target- pathway" network of RAS was constructed and the three metabolic pathways predicted by the network were consistent with the metabolism pathway of Angelica sinensis: Linoleic acid metabolism, Arginine and Proline metabolism, Tryptophan metabolism. The ALOX12B, ALOX12, ALOX15, ALOX15B, ALOX5 and PTGS1 in Linoleic acid metabolic pathway were regulated by ergapten, senkyunolideC, sedanolide and Thymol in Angelica active ingredient. The CYP1B1, CYP1A1 and CYP1A2 in Tryptophan metabolism were regulated by bergapten, Isoamylbenzene, beta-Chamigrene, Stigmasterol, Scopoletol and (3S)-3-butyl-3H-isobenzofuran-1-one. Therefore, the underlying 
mechanisms of action of RAS on hemolytic blood deficiency may be related to stabilization of the blood cell membrane, amino acids balance and regulation of the linoleic acid metabolism.

In conclusion, the proximate values and presence of RAS were very clear, suggesting that the studied Chinese herb has beneficial effects on improving hemolytic anemia and general well-being. In this study, we successfully applied metabolomics based on UPLC-Q-TOF/MS in the detection of key biomarkers and pathways in a rat model of hemolytic blood deficiency using RAS treatment. Differentially expressed metabolites were identified by metlin database (https://metlin.scripps.edu/), SIMCA software, MATPA, and KEGG analysis. The data presented in this study revealed that RAS possessed anti-anemic properties. Moreover, the effect of enriching blood using RAS may be related to the normalization of linoleic acid, l-glutamine, succinic acid, cytidine, cytosine, uracil, deoxycytidine, orotic acid, uridine, arginine, tryptophan. The results of multi-angle studies of pharmacodynamics, metabolomics, and network pharmacology support the scientific nature of traditional medicine theory and understanding of the blood nourishing mechanisms by the Angelica sinensis. However, the study was limited to the mentioned procedure and did not reports the data of standard compounds by TOF measurement procedure to report the amount of the substances that was identified in the extracts, analytically.

\section{Acknowledgments}

This study was supported by the National Natural Science Foundation of China (No. 81360633), Gansu Provincial Education Department Fund (2017A-056), Gansu Provincial Administration of Traditional Chinese Medicine Fund (GZK-2016-25), Open Fund Project of Key Laboratory of Molecular Medicine and Traditional Chinese Medicine Prevention and Control of Major Diseases in Colleges and Universities of Gansu Province (FZYX17-18-4) and Dunhuang School of Medicine and Translation Key Laboratory Open Fund Project (No:DHYX18-11). Natural Science Foundation of Gansu Province (18JR3RA201, 20JR10RA331). The funding agencies did not have any role in the study design, data collection and analyses, decision on publication, or preparation of the manuscript.

\section{ORCID}

Xiujuan Yang: 0000-0002-2608-3073

Yi Deng: 0000-0003-2645-4038

Zhijun Yang: 0000-0002-4560-6829

Shuo Li: 0000-0001-6645-4601

Yunxiang Hai: 0000-0001-9359-0801

Yihong Tian: 0000-0002-0508-3891

\section{References}

[1] L.E. Böttiger and B. Böttiger (1981). Incidence and cause of aplastic anemia, hemolytic anemia, agranulocytosis and thrombocytopenia, Acta Medica Scand. 210, 475-479.

[2] G. Benzi, G.L. Corona and G.M. Frico (1964). Effect of cobalt on anemia caused by acetylphenylhydrazine. Research in normal rats, Boll. Soc. Ital. Biol. Sper. 40, 1289-1292.

[3] Pharmacopeia of People's Republic of China (2015). Chinese Medicine and Pharmaceutical Publisher, Beijing, 133.

[4] M. Zhang, Y. Deng and Y. Wand (2014). The influence on peripheral blood routine, liver, spleen and thymus indexes of losing blood anemia mice of Angelica sinensis from Gansu province, Trad. Chin. Med. Res. 27, 69-71

[5] Y. Deng, M. Zhang and Y.P. Zhang, Y P. (2015). The intervention of Gansu DangGui on the mice models with acetyl phenyl hydrazine-induced hemolytic blood-deficiency, Western J. Trad. Chin. Med. 28, 5-8.

[6] J.K. Nicholson, J. Conrlelly and J.C. Liladorl (2002). Metabonomics: aplatformfor studying drug toxicity and gene function, Nat. Rev. Drug. Discov. 1(20), 153-161.

[7] W.B. Dunn, N.J.C. Bailey and H.E. Johnson (2005). Measuring the metabolome, Current. Anal. Technol. 30, 606-625. 
[8] J.K. Nicholson, J.C. Lindon and E. Holmes (1999). Metabonomics': Understanding the metabolic responses of living systems to pathophysiological stimuli via multivariate statistical analysis of biological NMR spectroscopic data, Xenobiotica 29(11), 1181-1189.

[9] X.J. Yang, Y.Z. Yang and Z.J. Yang (2017). Research progress on the application metabonomics in blood deficiency and blood stasis syndrome, Chin. J. Clin. Pharmacol. 33(11), 101-104.

[10] T. Ogura, T. Bamba and E. Fukusaki (2013). Development of a practical metabolite identification technique for non-targeted metabolomics, J. Chromatogr A. 1301, 73-79.

[11] G.W. Xu, X. Lu and S.L. Yang (2013). Recent advances in metabonomics, Acta Acad. Med. Sinic. 29(6), 701-711.

[12] Q. Chen (2013). The research and method of parmacology of traditional Chinese medicine. $2^{\text {nd }}$ edition. Beijing: People's Medical Publishing House. 90-503.

[13] Y.W. Wang, L. Qiao, C.F. Liu and N. Lin (2010). Effect and its mechanism of Coptis chinensis on oxidative hemolysis of erythrocytes in mice induced by acetylphenylhydrazine, China J. Chin. Mat. Med. 35, 2449-2452.

[14] F.T. Li, J.A. Duan and D.W. (2015). Effect of Elaphuri davidiani Cornu on the blood-deficiency model induced by acetylphenylhydrazine and cyclophosphamide in mice, Pharmacol. Clin. Chin. Mat. Med. 31, $100-103$.

[15] J. Xia and D.S. Wishart (2011). Web-based inference of biological patterns, functions and pathways from metabolomic data using MetaboAnalyst, Nat. Protoc. 6, 743-60.

[16] S. Bijlsma, I. Bobeldijk, E.R. Verheij, R. Ramaker, S. Kochhar, I.A. Macdonald, B. van Ommen and A.K. Smilde (2006). Large-scale human metabolomics studies: a strategy for data pre-processing and validation, Anal. Chem. 78, 567-574.

[17] T. Sangster, H. Major, R. Plumb, A. J. Wilson and I. D. Wilson (2006). A pragmatic and readily implemented quality control strategy for HPLC-MS and GC-MS based metabonomic analysis, Analyst 131(10), 1075-1078.

[18] X. Wang, H. Sun, A., Zhang, P. Wang and Y. Han (2011). Ultra-performance liquid chromatography coupled to mass spectrometry as a sensitive and powerful technology for metabolomic studies, J. Sep. Sci. 34, 3451-3459.

[19] T. Lundstedt, E. Seifert, L. Abramo, E. Seifert, B. Thelin, A. Nystrom, J. Pettersen and R. Bergman (1998). Experimental design and optimization, Chemom. Intell. Lab. Syst. 42, 3-40.

[20] M. Kanehisa and S. Goto (2012). KEGG for integration and interpretation of large-scale molecular data sets, Nucleic Acid. Res. 40, 109-114.

[21] J. Ru, P. Li, J. Wang. W. Zhou, B. Li, C. Huang, P. Li, Z. Guo, W. Tao, Y. Yang, X. Xu, Y. Li, Y. Wang and L. Yang (2014). TCMSP: a database of systems pharmacology for drug discovery from herbal medicines, Journal of Cheminformatics. 6, 13 (Six pages).

[22] X. Liu, J. Wu, D. Zhang, K. Wang, X. Duan and X. Zhang (2018). A network pharmacology approach to uncover the multiple mechanisms of Hedyotis diffusa Willd on colorectal cancer, Evid. Based Complement. Alternat. Med. e CAM. 4, 1-12.

[23] Muhammed J, Khan A, Ali A, L. Fand, Y. Wand, Q. Xu and D.Q. Wei (2018). Network pharmacology: exploring the resources and methodologies, Curr. Top. Med. Chem. 12(2), 121-30.

[24] E.J. Kanfer and B.A. Nicol (1997). Haemoglobin concentration and erythrocyte sedimentation rate in primary care patients, J. R. Soc. Med. 90, 16-18.

[25] B.S. Bull and J. Breton-Gorius (1995). Morphology of the erythron, Williams Hematology, ed. Ernest Beutler, William Joseph Williams, McGraw-Hill, Health Professions Division, pp. 349-352.

[26] Y. Kim, P. Joonhong and K. Myungshin (2017). Diagnostic approaches for inherited hemolytic anemia in the genetic era, Blood Res. 52(2), 84-94.

[27] X.Y. Shi and W.W. Gu (2008). Animal model of sickness in human, 1st edition. Beijing: People's Medical Publishine House. pp.242-243.

[28] M.S. Miao, L.P. Zhang, X.Y. Fang and Y.H. Sun (2002). The effect of Danggui buxue Tang on blood deficiency model mice induced by APH and CTX, China Basic. Med. J. Trad. Chin. Med. 8, 926-927.

[29] Chinese Pharmacopoeia Commission, Pharmacopoeia of the People's Republic of China, Chinese Medicine and Pharmaceutical Publisher. 2015, 133, Beijing

[30] J.M. Guo (2015). The research of medical Angelica sinensis on herbalism and pharmacognostics, J. China Prescript. Drug. 13, 18-19.

[31] X.H. Feng and X.L. Liang (2012). The research progress of chemical components and pharmacologic action in Angelica sinensis, Guangzhou Chem. Ind. J. 40, 16-18.

[32] X. Li, L.H. Zhang and X.X. Wang (2013). The research progress of chemical components and pharmacologic action in Angelica sinensis, J. Chin. Med. Mat. 36, 1023-1028.

[33] H. Yin (20150. The research progress of chemical components and pharmacologica activity in Angelica sinensis, J. Chongqing Univ. Sci. Technol. (Social Sciences Edition). 17, 100-101. 
[34] Q.Z. Shi, Z.L. Xu and J.S. Luo (20040. Experimental research of Danggui injection on aplastic anemia, Med. J. Wuhan Univ. 25, 557-559.

[35] X.T. Wu (1992). The abnormalities of nucleotide metabolic in red nucleus and hemolytic anemia, Foreign Blood Transfus. Hematol. 15(2), 85-87.

[36] M.B. Marrero, R.C. Venema, H. Ma, B.N. Ling and D.C. Eaton (1998). Erythropoietin receptor-operated Ca2q channels: activation by phospholipase C-gamma 1, Kidney Int. 53, 1259-1268.

[37] M.S. Chen and X.Q. Sheng (2007). Effects of $\alpha$-ketone acid and low protein diet on metabolism and nutritional status of chronic renal failure, Zhejiang Clin. Med. J. 9, 205-209.

[38] J. Wang, Y. Ruan and Q.Y. Tan (2008). The effect of $\alpha$-ketone acid on the treatment of early 2 diabetic nephropathy, J. Southeast China Nation. Defence Med. 10, 426-429.

[39] S. Stancheva, L. Alova and M. Stefanova (2009). Effect of peptide and nonpeptide antagonists of angiotensin II receptors on noradrenaline release in hypothalamus of rats with angiotensin II-induced increase of water intake, Pharmacol. Rep. 61(6), 1206-1210.

[40] E. Won and Y.K. Kim (2016). Stress, the autonomic nervous system, and the immune-kynurenine pathway in the etiology of depression, Curr. Neuropharmacol. 14(7), 665-673.

[41] P. Li, Y.L. Yin, D. Li, S.W. Kim and G. Wu (2007). Amino acids and immune function, Br. J. Nutr. 98, 237-252.

[42] D. Iin, S. Kim and S. Park (2004). Hexokinase method can be used to determine the hypotheality of blood glucose in some patients with anemia, Med. Lab. 19 (4), 119-135.

[43] Y.R. Du and Y.L. Zhao (2008). The relationship between erythrocyte biochemical characteristics and morphological function. Hebei Med. J. 30(2), 216-217.

[44] Q.Y. Xu, C.A. Wang and H. Xu (2009). The effect of propiony l-glutamine on the growth and antioxidant capacity of lara fish, Chin. J. Animal Nutr. 21(6), 1012-1017.

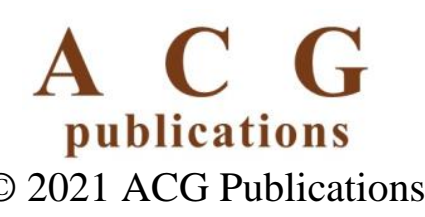

